\title{
Measuring the 3D Point Spread Function of Super- resolving Pupil Filters Focused into a Refractive Medium
}

\author{
D. Iwaniuk, E. Hack \\ Laboratory of Electronics/Metrology/Reliability, Empa, Überlandstr. 129, 8600 Dübendorf, Switzerland \\ daniel.iwaniuk@empa.ch
}

\begin{abstract}
The focal spot size of superresolution pupil filters is measured with a new technique based on a fluorescent screen for imaging through air and a refractive index mismatch. It allows to measure high NA lenses.

(C)2009 Optical Society of America

OCIS codes: (110.0180) Microscopy; (100.6640) Superresolution; (120.5060) Phase modulation; (050.1970) Diffractive optics; (080.0080) Aberrations; (230.3720) Liquid-crystal devices.
\end{abstract}

\section{Introduction}

The theory of superresolution pupil filters has been investigated thoroughly in the last decades. Most simulations in the literature base on the formulations summarized by Goodman [1]. First realizations of superresolution were based on pupil filters with amplitude modulation. Meanwhile, phase-only and hybrid pupil filters have taken the lead due to higher light efficiency and the development of easy to use liquid crystal spatial light modulators. Although the applications vary from optical data storage, astronomy, and optical fiber communication to optical tweezers, the main research aims to improve optical light microscopes. The most popular filters are annular two and three zone binary phase filters, as they can be designed analytically within the second order approximation [2-4].

In spite of the huge investigations in theoretical simulations, there exists only little experimental verification [58]. They are all realized with low numerical apertures (NA) less than NA $=0.046$ [5-7]. To be useful for microscopy, higher NA must be examined. A new technique for measuring the focusing properties of lenses and superresolution pupil filters combined with lenses is presented. The main idea is to screen the point spread function (PSF) on a 40-50 nm thick fluorescent layer and to magnify it with a microscope objective with higher numerical aperture. Experimental proof of principle is given with $\mathrm{NA}=0.05$, but this method allows to measure lenses with much higher NA.

Furthermore, a planar refractive index mismatch occurs often in microscopy, may it be from a cover glass, immersion objective, specimen-mounting medium or a thick sample. D. Jiang and J.J. Stamnes [8] have shown that the primary effects are a focal shift and spherical aberrations, depending on the propagation length. Experiments were done which focus into uniaxial crystals with a NA of only 0.027 . To our knowledge, nobody performed experiments with superresolution for focusing into a medium. The effects of aberration and refractive index mismatch combined with high NA must be controlled before superresolution becomes a standard method in life cell microscopy.

We model a planar refractive index mismatch by focusing the beam through a glass plate onto the fluorescent layer. Aberration effects decreasing the efficiency of the superresolution pupil filter are shown. Measurements show the decrease of focal spot size in 3D by applying an annular three zone binary phase superresolution filter.

\section{Design and simulations of annular 3 zone binary phase-only superresolution filters}

The light intensity near focus can be calculated by equation 1 [1]:

$$
I(r, z)=\left|\frac{k}{i z} \cdot \int_{0}^{1} P(\rho) \cdot e^{\frac{-i k \rho^{2}}{2 f}} \cdot e^{\frac{i k \rho^{2}}{2 z}} \cdot J_{0}\left(\frac{k}{f} \rho r\right) \cdot \rho d \rho\right|^{2}
$$

The pupil plane is assumed to have radial symmetry with coordinate $\rho$, while the radial coordinate near focus is $r$. The longitudinal coordinate $z$ is the distance to the pupil plane. $k$ is the wave number of the illumination, $f$ the focal length of the lens, $J_{0}$ the zero order Bessel function and $P(\rho)$ is the so called pupil function. For a phase only pupil, $|P(\rho)|$ is equal to 1 . To generate superresolution in axial and transversal direction (3D superresolution), Sheppard [2] showed that annular three zone binary phase-only filters $P(\rho)=e^{i \varphi(\rho)}$ can be used, where $\varphi(\rho)$ is 0 for the inner and outer zone and $\pi$ in the middle zone. The pure phase 3D (P3D) filter from table 1 of reference [2] was used in this work with normalized radii $\rho_{I}=0.48, \rho_{2}=0.64$. Simulations based on equation 1 give an axial gain $G_{A}=$ 1.45 , a transversal gain $G_{T}=1.17$ and a Strehl ratio of $S=0.3$ when focusing through air. 


\section{Experimental set-up for measuring the point spread function (PSF)}

The experimental set-up is shown in figure 1. An expanded laser beam (CrystaLaser GCL-050-S, 532 nm, 50mW) with aperture $10 \mathrm{~mm}$ is modulated by a phase-only spatial light modulator in reflection (Holoeye Pluto SLM, LCOS, 1920x1080, $8 \mu \mathrm{m}$ pitch). The calibration of the phase-only SLM was done with a Michelson interferometer. The modulated light from the SLM is focused by a singlet lens (Newport KBX052, $50.2 \mathrm{~mm}$ focal length) on a fluorescent screen (1-ethyl-2-((1E,3E)-3-(1-ethyl-3,3-dimethylindolin-2-ylidene)prop-1-enyl)-3,3-dimethyl-3Hindolium perchlorate) dye (CY5) spin coated to a thickness of $40-50 \mathrm{~nm}$ on a $5 \mathrm{~mm}$ thick glass plate with refractive index $\mathrm{n}=1.5$. A microscope objective (e.g. 10x NA =0.25) images the spot onto the camera (Prosilica GC1600, 1/1.8" CCD, 1600x1200, $4.4 \mu \mathrm{m}$ pixels), while a colour filter (Newport OG550) suppresses the laser illumination light and transmits the red-shifted fluorescent light. The whole imaging system can be translated with a linear stage (Newport GTS150) with $0.1 \mu \mathrm{m}$ resolution.

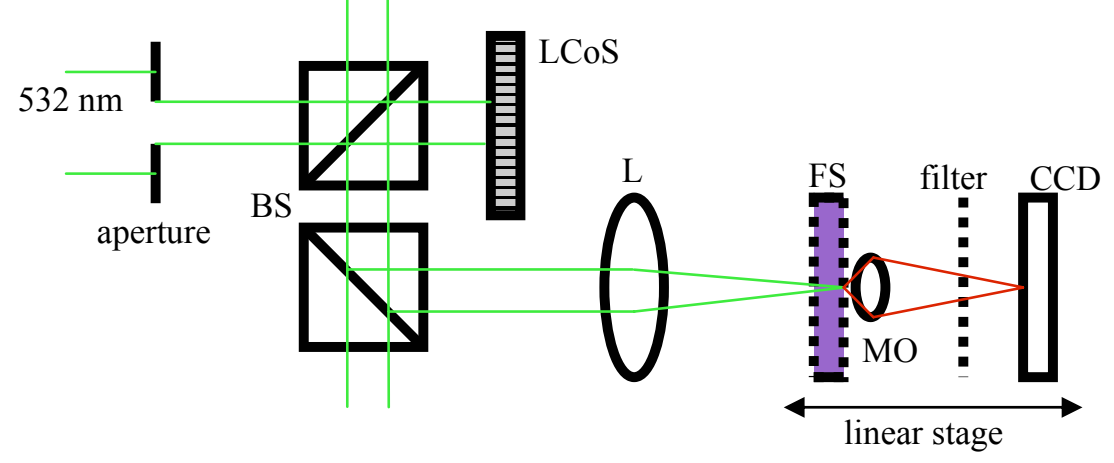

Fig. 1. Measurement setup to visualize the focusing spot of a lens modulated with a phase function induced by a spatial light modulator. (BS: 50:50 beam splitters, LCoS: liquid crystal on silicon, CCD: camera, L: lens, FS: fluorescent screen on either side of glass plate, MO: microscope objective).

This measurement system has several benefits. Displaying the focal spot on the fluorescent screen gives directly its actual PSF without errors from propagation or "out-of-focus" light. By choosing on which side of the glass the fluorescent layer is coated, the light passes either solely through air or through the glass, which represents a refractive index mismatch. The calibration of the lateral resolution was done with a 40 line pairs per mm grating placed on the same side of the glass as the fluorescent dye, resulting in around 3 pixels per micrometer.
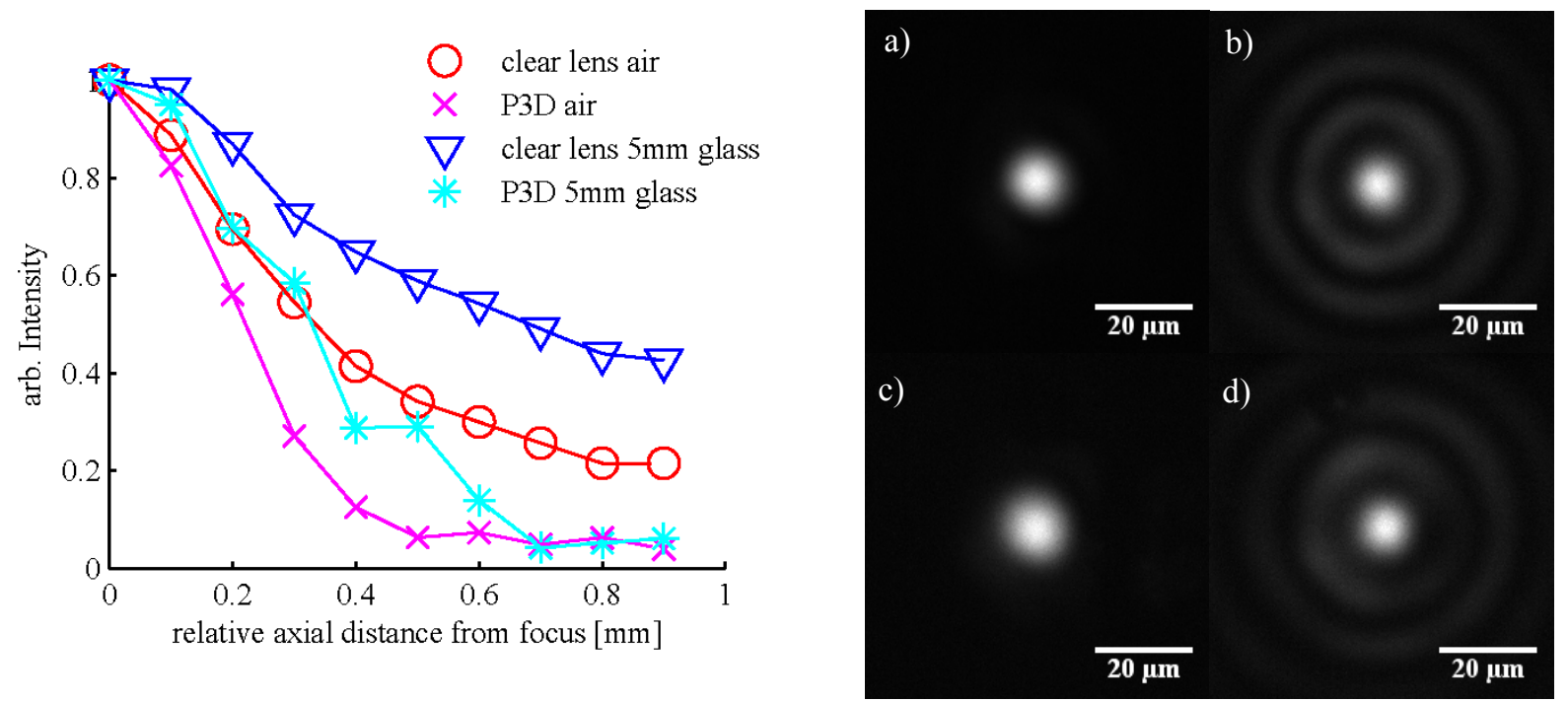

Fig. 2. PSF measurements in axial direction through focus (left) and in transversal direction in focus (right) (a: clear lens through air, b: P3D through air, c: clear lens through glass, d: P3D through glass).

The measurements of the three-dimensional PSF are shown in figure 2. The axial measurement was done by starting out of focus and then translating the fluorescent screen through focus. Care must be taken at the high intensities in focus, as the colour dye bleaches quite fast. The transversal PSF were measured in focal position. The full width at half maximum (FWHM) in axial and transversal directions are shown in table 1 together with the 


\section{NWB4.pdf}

performed simulation. The simulation represents the diffraction limit, while the Newport singlet lens shows spherical aberrations as expected. However, the axial and transversal gain for focusing through air agrees quite well with the simulation.

Table 1. Simulations and experimental results. $\left(\mathrm{G}_{\mathrm{A}}\right.$ : axial gain, $\mathrm{G}_{\mathrm{T}}$ : transversal gain $)$

\begin{tabular}{|c|c|c|c|c|c|c|}
\hline \multirow{2}{*}{} & \multicolumn{2}{|c|}{ simulation } & \multicolumn{4}{c|}{ experiment } \\
\cline { 2 - 7 } & \multicolumn{2}{|c|}{ air } & \multicolumn{2}{c|}{ air } & \multicolumn{2}{c|}{5 mm glass } \\
\cline { 2 - 7 } & clear & P3D & clear & P3D & clear & P3D \\
\hline axial FWHM $[\mathrm{mm}]$ & 0.376 & 0.26 & 0.68 & 0.46 & 1.38 & 0.66 \\
\hline $\mathrm{G}_{\mathrm{A}}$ & & 1.45 & & 1.5 & & 2.1 \\
\hline transversal FWHM $[\mu \mathrm{m}]$ & 5.44 & 4.64 & 8.93 & 8.56 & 10.4 & 8.56 \\
\hline $\mathrm{G}_{\mathrm{T}}$ & & 1.17 & & 1.04 & & 1.27 \\
\hline
\end{tabular}

Focusing through glass of thickness $5 \mathrm{~mm}$ introduces severe aberrations compared to the results in air. The axial width of the PSF of the clear aperture is doubled. The superresolution pupil filter was able to restore the values of the axial and transversal FWHM of the clear aperture in air.

\section{Conclusions}

We have presented a method to measure the point spread function directly on a thin fluorescent layer. This allows to assess experimentally the performance of all kind of pupil filters such as superresolution filters or filters for adaptive wavefront correction. The advantage of the method is its high luminosity as compared to the observation of a single fluorescent sub-wavelength bead. Future work will provide measurements for higher NA and simulations for the refractive index mismatch. Furthermore, an adaptive correction of the focus shift and spherical aberrations will be done.

The authors would like to thank the Swiss National Science Foundation (SNSF) for financial support and S. Kuster (EMPA, Laboratory for Functional Polymers) for providing the fluorescent dye and coating the glass plates.

\section{References}

[1] J.W. Goodman, Introduction to Fourier optics (McGraw-Hill, $2^{\text {nd }}$ Ed. 1996).

[2] C.J.R. Sheppard, J. Campos, J.C. Escalera, S. Ledesma, “Three-zone pupil filters”, Opt Commun 281, 3623-3630 (2008).

[3] X. Gao, F. Gan, W. Xu, "Superresolution by three-zone pure phase plate with 0, $\pi$, 0 phase variation", Opt \& Laser Technol 39, 1074-1080 (2007).

[4] V.F. Canales, J.E. Oti, M.P. Cagigal, “Three-dimensional control of the focal light intensity distribution by analytically designed phase masks", OptCommun 247, 11-18 (2005).

[5] E. Ramsay, K.A. Serrels, A.J. Waddie, M.R. Taghizadeh, D.T. Reid, “Optical super-resolution with aperture-function engineering”, Am J Phys 76, 1002-1006 (2008).

[6] P.N. Gundu, E. Hack, P.K. Rastogi, "High efficient superresolution combination filter with twin LCD spatial light modulators", Opt Express 13, 2835-2842 (2005).

[7] H. Luo, C. Zhou, “Comparison of superresolution effects with annular phase and amplitude filters”, Appl Opt 43, 6242-6247 (2004).

[8] D. Jiang, J.J. Stamnes, "Numerical and experimental results for focusing of two-dimensional electromagnetic waves into uniaxial crystals", Opt Commun 174, 321-334 (2000). 